ISSN 0103-5150

Fisioter. Mov., Curitiba, v. 26, n. 1, p. 47-53, jan./mar. 2013

Licenciado sob uma Licença Creative Commons

\title{
Locomoção, distúrbios neuropsiquiátricos e alterações do sono de pacientes com demência e seus cuidadores
}

\author{
Locomotion, neuropsychiatric disturbs and sleep disorder \\ in patients with dementia and their caregivers
}

\author{
Gustavo Christofoletti $^{[\mathrm{a}]}$, Rodrigo Luiz Carregaro ${ }^{[\mathrm{b}]}$, Merlyn Mércia Oliani ${ }^{[\mathrm{cl}}$, Florindo Stella ${ }^{[\mathrm{d}]}$, \\ Lilian Teresa Bucken-Gobbi ${ }^{[e]}$, Sebastião Gobbi ${ }^{[f]}$
}

[a] Doutor em Ciências Biomédicas pela Universidade Estadual de Campinas (Unicamp), docente do Centro de Ciências Biológicas e da Saúde e do Programa de Pós-Graduação em Saúde e Desenvolvimento do Centro-Oeste da Universidade Federal de Mato Grosso do Sul (UFMS), Campo Grande, MS - Brasil, e-mail: gustavo_physio@yahoo.com.br

[b] Doutor em Ciências da Saúde pela Universidade de Brasília (UnB), docente do curso de Fisioterapia e do Programa de Pós-Graduação em Educação Física (UnB), Brasília, DF - Brasil, e-mail: rodrigocarregaro@unb.br

[c] Mestre em Ciências da Motricidade Humana pela Universidade Estadual Paulista Júlio de Mesquita Filho (Unesp), coordenadora do curso de Educação Física da Faculdade Unifadra (Unifadra), Dracena, SP - Brasil, e-mail: meloliani@yahoo.com.br

[d] Livre-docente pelo Instituto de Biociências da Universidade Estadual Paulista (Unesp), docente do Departamento de Educação e do Programa de Pós-Graduação em Ciências da Motricidade Humana da Universidade Estadual Paulista (Unesp), Rio Claro, SP - Brasil, e-mail: fstella@rc.unesp.br

[e] Livre-docente pelo Instituto de Biociências da Universidade Estadual Paulista (Unesp), docente do Departamento de Educação Física, coordenadora do Programa de Pós-Graduação em Ciências da Motricidade da Universidade Estadual Paulista (Unesp), Rio Claro, SP - Brasil, e-mail: ltbgobbi@rc.unesp.br

[f] Livre-docente pelo Instituto de Biociências da Universidade Estadual Paulista (Unesp), docente do Departamento de Educação Física e do Programa de Pós-Graduação em Ciências da Motricidade Humana da Universidade Estadual Paulista (Unesp), Rio Claro, SP - Brasil, e-mail: sgobbi@rc.unesp.br

\section{Resumo}

Introdução: Os distúrbios neurodegenerativos representam condições clínicas graves, por provocar declínio neuropsíquico. Objetivo: Analisar a prevalência dos distúrbios neuropsiquiátricos em pacientes com demência, em relação à sua locomoção (independentes vs. dependentes), e no que se refere ao desgaste emocional e à qualidade do sono dos cuidadores. Materiais e métodos: Participaram do estudo 34 sujeitos, assim divididos: dez pacientes independentes para locomoção e sete dependentes (cadeirantes); dez cuidadores dos pacientes independentes e sete cuidadores de pacientes dependentes. Os sujeitos foram avaliados no Ambulatório 
de Neuropsiquiatria da Universidade Estadual de Campinas. Para avaliar as funções cognitivas, utilizou-se o CAMCOOG; para quantificar frequência, intensidade e desgaste do cuidador, aplicou-se o Inventário Neuropsiquiátrico; e para mensurar as alterações do sono dos cuidadores, foi utilizado o Miniquestionário do Sono. A análise estatística foi realizada por meio dos testes $U$ - Mann Whitney e índice de correlação de Spearman, ambos com 5\% de significância. Resultados: Com relação à prevalência dos distúrbios neuropsiquiátricos entre os pacientes, observou-se diferença estatisticamente significativa entre os grupos, especificamente no que se refere à irritabilidade $(\mathrm{p}<0,05)$ e ao escore total dos distúrbios neuropsiquiátricos $(p<0,01)$. Também foram encontradas diferenças entre os grupos de cuidadores, com relação às alterações do sono $(\mathrm{p}<0,05)$. Conclusão: Idosos independentes para locomoção apresentam menor prevalência dos distúrbios neuropsiquiátricos, quando comparados a idosos dependentes de cadeira de rodas. A locomoção parece não influenciar no desgaste físico e emocional do cuidador, mas constitui uma variável relevante na qualidade do sono dos cuidadores de idosos com diagnóstico de demência vascular e mista.

Palavras-chave: Demência. Locomoção. Distúrbios neuropsiquiátricos. Alterações no sono.

\section{Abstract}

Introduction: The neurodegenerative disorders represent serious clinical conditions, causing neuropsychiatric decline. Objective: To evaluate the prevalence of neuropsychiatric disorders in subjects with dementia, with respect to their locomotion (independent versus dependent ones) and to the caregivers' distress and sleep quality. Materials and methods: Thirty-four subjects participated in this study: ten independent locomotion patients and seven dependent ones (using wheelchair); ten carers of the independent locomotion patients and seven related to those that use wheelchairs. The subjects were recruited in the Clinic of Neurosciences and Mental Health of the Elderly of the State University of Campinas. The CAMCOG was used to evaluate patients' cognitive function; in order to quantify the frequency, intensity, emotional and physical distress of caregivers, the Neuropsychiatric Inventory was applied. Changes in the sleep were evaluated by means of Mini-Sleep Questionnaire. For comparisons of scores between the instruments, the Mann Whitney U and the Spearman coefficients index were applied, with a significant level of 5\%. Results: There were differences among irritability disorders ( $p<0.05)$ as well as the total score of frequency and intensity of neuropsychiatric disorders $(p<0.01)$. Still, there were differences between the caregivers groups, in respect to sleep disorders ( $p<0.05)$. Conclusion: Independent locomotion patients presented a lower prevalence of neuropsychiatric disorders, compared to the dependent ones. Findings suggest that motion does not influence the level of physical and emotional exhaustion of the caregiver. Nevertheless, it can be considered a relevant variable for sleep quality.

Keywords: Dementia. Locomotion. Neuropsychiatric disturbs. Sleep disorders.

\section{Introdução}

0 fato de os seres humanos serem bípedes e de se locomoverem com fases de apoio unipodal, sem nenhum contato (corrida), ou permanecer parado com o contato de ambos os pés (ortostatismo), cria um desafio adaptativo aos sistemas que controlam o equilíbrio, tornando-se necessárias informações contínuas sobre a posição e o movimento de todas as partes do corpo (1). Tal controle também requer processamentos cognitivos e motores, cujos mecanismos envolvidos estão relacionados aos recursos atencionais, funções executivas e aos sistemas sensorial e musculoesquelético (2). Assim, a locomoção depende da funcionalidade desses processos sob a regência de estruturas cerebrais superiores, comumente comprometidas nas demências (3).

De forma geral, os distúrbios neuropsiquiátricos manifestados em pacientes com demência estão associados a etiologia, alterações bioquímicas, fatores genéticos e psicossociais (4). Nas fases iniciais da demência, os distúrbios neuropsiquiátricos mais prevalentes e clinicamente mais relevantes são depressão, apatia, ansiedade e irritabilidade. Com a progressão da demência, o quadro neuropsiquiátrico agrava-se, surgindo sintomas psicóticos, agitação, 
distúrbios do sono, comportamentos bizarros e acentuação da apatia. 0 agravamento neuropsiquiátrico tende a acompanhar progressão da atrofia, que passa a afetar todo o funcionamento cerebral (5). Contudo, a manifestação também pode ser desencadeada em função de uma reação sintomática, geralmente ligada a processos infecciosos gastrintestinal, geniturinário, oftalmológico, otolaringológico ou respiratório (6).

O termo "demência vascular" (DV) tem conotações amplas, referindo-se a qualquer demência causada por doença cerebrovascular, como infarto simples, múltiplos infartos corticais e lacunares, alterações crônicas da circulação cerebral, doença Binswanger, leucoaraiose, angiopatia amiloide e acidente cerebral encefálico hemorrágico (7). Segundo os critérios diagnósticos do DSM-IV (8) e NINCDS-AIREN (9), a deteriorização nessa demência é abrupta ou em escada, decorrente de um quadro confusional no período de três meses após a lesão cerebral. Os exames de neuroimagem são de suma importância para o diagnóstico, podendo mensurar localização, tamanho, distribuição unilateral ou bilateral e quantidade de lesões; áreas como hipocampo, tálamo e núcleo caudado indicam maiores comprometimentos cognitivos (10).

A presença de lesões cerebrovasculares em pacientes com demência de Alzheimer caracteriza uma entidade nosológica chamada de demência mista (DM), muitas vezes subestimada (11). 0 diagnóstico da DM deve ser realizado na presença de uma (ou mais) doença sistêmica ou cerebral, relacionada etiologicamente à demência de Alzheimer ou vascular (12). De acordo com estudos patológicos, estima-se que mais de um terço dos pacientes com demência de Alzheimer apresenta também lesões vasculares, e proporções similares podem ser encontradas em pacientes com diagnostico de DV, com características de Alzheimer (13).

As demências são patologias que acometem a independência e a autonomia do paciente demandando do cuidado de terceiros que, em geral, são membros da própria família, maioria mulheres que moram com o paciente. 0 processo de cuidar envolve aspectos emocionais, sociais e físicos, e fatores como idade e nível intelectual do cuidador também são relevantes durante o acompanhamento e a assistência ao paciente demenciado (14). Por conta da alta carga de trabalho, são comuns problemas fisiológicos e psicológicos nos cuidadores, sobretudo relacionados à baixa imunidade celular, respostas arteriais elevadas, depressão, ansiedade, estresse e alterações do sono (15).

0 objetivo deste estudo foi analisar a prevalência de distúrbios neuropsiquiátricos em pacientes com demência, em relação à sua locomoção (independente versus dependente); e verificar a influência da locomoção no desgaste emocional e na qualidade do sono de cuidadores dos pacientes.

\section{Materiais e métodos}

\section{Tipo de estudo}

Este estudo, com delineamento transversal, investigou 34 idosos de ambos os sexos, com diagnóstico de DV e DM, de acordo com os critérios estabelecidos pelo DSM-IV (8) e NINCDS-AIREN (9), e seus respectivos cuidadores.

\section{Sujeitos}

A amostra foi composta por 17 pacientes, sendo dez idosos independentes para locomoção (Grupo A - GA) e sete idosos dependentes de cadeira de rodas (Grupo B - GB); e dez cuidadores de pacientes independentes $\left(\mathrm{GA}_{1}\right)$ e sete cuidadores de pacientes dependentes de cadeira de rodas $\left(\mathrm{GB}_{1}\right)$. Os sujeitos foram recrutados no Ambulatório de Neuropsiquiatria e Saúde Mental do Idoso, do Hospital das Clínicas da Universidade Estadual de Campinas (Unicamp) e a coleta de dados foi realizada no momento da consulta do paciente.

Exames complementares de neuroimagem foram realizados para confirmação diagnóstica (tomografia computadorizada, ressonância magnética e tomografia computada por emissão de fóton único). Os principais marcadores fisiológicos e anatomopatológicos da amostra foram evidenciados por hipersinal de substância branca periventricular, especialmente nas áreas frontal, temporal e occipital; hipoperfusão dos lobos frontais; leucoaraiose difusa, dilatação dos sulcos e ventrículos, vasculopatia da substância branca, e atrofia frontal, temporoparietal e parieto-occipital. Os psicofármacos mais utilizados pelos pacientes consistiam dos inibidores da enzina acetilcolinesterase, antiglutamatérgicos, antidepressivos, ansiolíticos, neurolépticos, vasodilatadores e polivitamínicos. 
O estudo foi aprovado pelo Comitê de Ética em Pesquisa da Universidade Estadual Paulista Unesp/Rio Claro. Todos os participantes assinaram um Termo de Consentimento Livre e Esclarecido, baseado na Resolução n. 196/96 do Ministério da Saúde.

\section{Procedimentos de avaliação}

Para mensurar as funções cognitivas do paciente, utilizou-se o CAMCOG, que corresponde a uma parte do Cambridge Examination for Mental Disorders of the Elderly (16). 0 instrumento divide-se em dez seções que investigam cada domínio cognitivo, como orientação, linguagem de compreensão e expressão, memória remota, memória recente, memória de aprendizagem, atenção, praxias, funções executivas e percepção. 0 escore varia de 0 a 105 , e pontuações elevadas indicam funções cognitivas preservadas.

O Inventário Neuropsiquiátrico (NPI) (17) foi utilizado para quantificar a ocorrência dos distúrbios comportamentais e outros transtornos mentais ocorridos nas últimas três semanas. O NPI é divido em dez domínios: delírios, alucinações, agitação, depressão e disforia, ansiedade, euforia e elação, apatia e indiferença, desinibição, irritabilidade e labilidade emocional, comportamento motor aberrante, comportamentos noturnos e alterações alimentares. Cada domínio é avaliado pela frequência (escores de 1 a 4), intensidade ( 1 a 3 ) e desgaste do cuidador ( 1 a 5 ). 0 escore total do instrumento possui uma amplitude de 0 a 120 para frequência e intensidade; e de 0 a 50 para a avaliação do desgaste do cuidador. Escores elevados representam maior frequência, intensidade e desgaste desses eventos.

Para avaliar a qualidade do sono dos cuidadores, utilizou-se o Miniquestionário do Sono (MQS) (18), instrumento que se divide em dez questões, com sete alternativas, as quais quantificam a ocorrência de alterações do sono por meio de escala de 1 a 7 pontos. 0 escore total é de 70 pontos, sendo que escores superiores a 30 representam sono muito comprometido. Todas as análises foram realizadas no Ambulatório de Neuropsiquiatria e Saúde Mental do Idoso do Hospital das Clínicas da Unicamp, em sala reservada, sob a supervisão de um docente psiquiatra que possui vasta experiência na referida área.

\section{Análise estatística}

Utilizou-se o programa SPSS versão 13.0, com significância de $5 \%(\mathrm{p}<0,05)$ em todas as análises. 0 procedimento estatístico consistiu na utilização da análise descritiva dos dados (média e desvio padrão). Além disso, utilizou-se o teste não paramétrico $U$ - Mann Whitney, para comparar os escores entre as funções cognitivas, os distúrbios neuropsiquiátricos e as alterações do sono dos grupos de pacientes e cuidadores. Também, utilizou-se o coeficiente de correlação de Spearman para observar a relação entre as variáveis.

\section{Resultados}

As características demográficas, clínicas, comportamentais e as alterações do sono encontram-se descritas no Quadro 1. Não foi observada diferença significativa nas variáveis: idade GA e GB (UMW $=26,5, \mathrm{p}>0,05)$, idade $\mathrm{GA}_{1}$ e $\mathrm{GA}_{2}$ (UMW = 29,5, p > 0,05); escolaridade GA e GB (UMW = $33,5, \mathrm{p}>0,05$ ), escolaridade $\mathrm{GA}_{1}$ e $\mathrm{GA}_{2}$ (UMW = 17,5, p < 0,05); gravidade da doença do GA e GB (UMW = 19,0, p > 0,05); tempo de doença (UMW = $24,5, \mathrm{p}>0,05)$; e funções cognitivas GA e GB (UMW=20,5; p > 0,05).

Quanto à prevalência dos distúrbios neuropsiquiátricos entre o GA e GB, observou-se uma diferença significante para os distúrbios de irritabilidade (UMW = $11,0, p<0,05$ ) e no escore total de frequência e intensidade dos distúrbios neuropsiquiátricos (UMW = 10,0, $\mathrm{p}<0,01$ ). 0 Gráfico 1 apresenta a média de ocorrência dos distúrbios entre GA e GB. Não foram apontadas diferenças significativas tanto com relação os outros distúrbios neuropsiquiátricos entre GA e GB, quanto para o nível de desgaste do cuidador no $\mathrm{GA}_{1}$ e $\mathrm{GB}_{2}$ $(p>0,05)$. Ainda, não houve diferença entre os grupos $\mathrm{GA}_{1}$ e GB $\mathrm{GB}_{2}$ com relação às alterações do sono (UMW = $14,0, p>0,05$ ).

Houve uma correlação de fraca magnitude entre as variáveis locomoção e funções cognitivas $\left(r_{s}=-0,35\right)$ e locomoção e alterações do sono do cuidador $\left(r_{s}=0,12\right)$. No entanto, para os distúrbios de irritabilidade $\left(r_{s}=0,62\right)$ e escore total de frequência e intensidade dos distúrbios neuropsiquiátricos do paciente $\left(r_{s}=0,61\right)$, foram observadas correlações de moderada magnitude. 
Quadro 1- Características demográficas e clínicas da amostra, distúrbios neuropsiquiátricos do paciente, nível de desgaste e alterações do sono do cuidador

\begin{tabular}{|c|c|c|c|c|}
\hline & \multicolumn{2}{|c|}{ Paciente } & \multicolumn{2}{|c|}{ Cuidador } \\
\hline & GA & GB & $\mathrm{GA}_{1}$ & $\mathrm{~GB}_{1}$ \\
\hline Tamanho amostral & 10 & 7 & 10 & 7 \\
\hline Idade (anos) & $74,7 \pm 2,2$ & $76,7 \pm 1,4$ & $46,7 \pm 5,5$ & $51,8 \pm 2,2$ \\
\hline Escolaridade (anos) & $1,2 \pm 0,5$ & $0,7 \pm 0,2$ & $9,6 \pm 1,2$ & $6,0 \pm 2,2$ \\
\hline CDR & $3,4 \pm 0,5$ & $4,7 \pm 0,1$ & NA & NA \\
\hline Tempo de doença (anos) & $8,3 \pm 2,5$ & $5,0 \pm 1,3$ & NA & NA \\
\hline Funções cognitivas & $36,4 \pm 7,6$ & $18,8 \pm 7,8$ & NA & NA \\
\hline Distúrbios neuropsiquiátricos & $36,3 \pm 6,1$ & $69,7 \pm 9,0$ & $16,0 \pm 2,1$ & $26,0 \pm 3,8$ \\
\hline Nível de atividade física & $0,31 \pm 0,1$ & $1,13 \pm 2,7$ & $2,5 \pm 0,4$ & $3,3 \pm 1,6$ \\
\hline Alteracões do sono & NA & NA & $28,5 \pm 2,7$ & $39,0 \pm 3,4$ \\
\hline
\end{tabular}

Legenda: $\mathrm{GA}=$ grupo de idosos independentes para locomoção; $\mathrm{GB}=$ grupo de idosos cadeirantes. $\mathrm{GA}_{1}=$ grupo de cuidadores de idosos independentes para locomoção; $\mathrm{GB}_{1}=$ grupo de cuidadores de idosos cadeirantes. CDR: nível de gravidade de demência; $\mathrm{NA}=$ não aplicável.

Fonte: Dados da pesquisa

Nota: Os dados foram dispostos em média e desvio padrão.

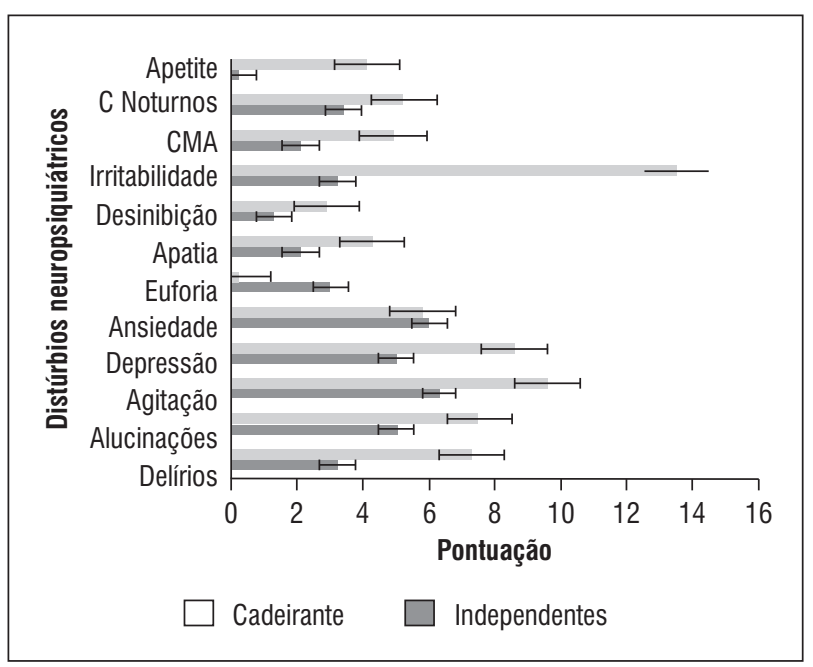

Gráfico 1 - Prevalência dos distúrbios neuropsiquiátricos dos idosos independentes e dependentes de cadeira de rodas para locomoção

Fonte: Dados da pesquisa.

Nota: Os dados foram dispostos em média e desvio padrão.

\section{Discussão}

Os dados desta pesquisa indicaram diferenças significativas entre a locomoção e a frequência e intensidade dos distúrbios neuropsiquiátricos, especialmente dos distúrbios de irritabilidade do paciente. Com relação às alterações do sono dos cuidadores, diferenças importantes foram evidenciadas entre os grupos, indicando que o padrão de sono do $\mathrm{GA}_{1}$ é levemente alterado, enquanto o $\mathrm{GB}_{2}$ classifica-se como muito alterado.

Segundo os dados apontados pelo coeficiente de Spearman, o índice de determinação da locomoção interfere em 38\% na frequência e intensidade dos eventos de irritabilidade e $37 \%$ na manifestação total de todos os distúrbios neuropsiquiátricos. O GA apresentou uma prevalência dos distúrbios de ansiedade, agitação e depressão, compatível com os dados revelados por meio dos exames de neuroimagens, que mostraram hipoperfusão cerebral, dilatação ventricular, lesão de substância branca e atrofia frontal e parietal. Tais alterações cerebrais e comportamentais corroboram com dados da literatura $(19,20)$.

Em relação ao distúrbio de irritabilidade para o grupo GB, houve grande prevalência, seguido de agitação e depressão. Os dados fisiológios e anatomopatológicos desses idosos demonstraram lesões de substância branca periventricular e difusas, alargamento de sulcos e ventrículos, além de hipoperfusão cerebral e cerebelar. Esses resultados sugerem uma investigação específica para se averiguar as possíveis relações entre tais marcadores fisiopatológicos e a prevalência dos distúrbios neuropsiquiátricos. 
Assim, a prevalência de tais distúrbios no GB parece associada a maiores comprometimentos cerebrais, sobretudo de substância branca. Estudos apontam as lesões de substância branca como indicativas de aceleração do declínio cognitivo e da manifestação de eventos psicóticos (21). Contudo, vale ressaltar que tal aspecto ainda permanece em aberto, sendo uma questão controversa na literatura, especialmente na DV e DM (22).

Sbin et al. (23) analisaram a influência dos distúrbios neuropsiquiátricos na qualidade de vida do paciente com demência e de seus respectivos cuidadores. Segundo os autores, a frequência e a intensidade dos distúrbios neuropsiquiátricos do paciente alteram negativamente em $20 \%$ a qualidade de vida do paciente e $73 \%$ a percepção de qualidade de vida do cuidador. Tal resultado torna-se ainda mais expressivo quando levamos em consideração que a manifestação deles constitui-se de aspectos multifatoriais.

O sono é uma atividade necessária aos processos fisiológicos responsáveis pelo equilíbrio físico e mental dos seres humanos. Analisando separadamente os dados com relação ao sono do cuidador, cerca de $14 \%$ das alterações do sono observadas foram decorrentes da condição de dependência dos pacientes. McCurry et al. (24) investigaram o sono de 46 cuidadores de idosos com demência por meio da técnica de actigrafia e perceberam que $88 \%$ das alterações do sono vivenciadas por cuidadores relacionam-se com o ciclo sono-vigilía do paciente, especialmente com eventos de perambulação.

A locomoção representa uma condição de independência importante, sobretudo em virtude da complexidade do ato, que depende da integridade das vias piramidais e extrapiramidais e de comandos cerebrais conscientes e inconscientes (25). Neste estudo, foi constatada relação inversa entre locomoção e funções cognitivas, indicando que quanto maior a dependência física, menor será o desempenho cognitivo.

De acordo com os resultados encontrados, a variável locomoção representa benefícios tanto em relação à prevalência dos distúrbios neuropsiquiátricos, quanto aos aspectos cognitivos de idosos com DV. Diante disso, a adoção de medidas não farmacológicas capazes de possibilitar a manutenção do aparelho locomotor em ambos os grupos faz-se necessária, assim como a multidisciplinaridade entre as ciências humanas pode atenuar os efeitos negativos derivados da restrição do ato de caminhar independentemente e dos declínios progressivos característicos das doenças demenciantes.
Em conclusão, os idosos independentes para locomoção apresentaram menor prevalência dos distúrbios neuropsiquiátricos, quando comparados a idosos dependentes de cadeira de rodas. A locomoção parece não influenciar o nível de desgaste físico e emocional do cuidador, porém trata-se de uma variável relevante na qualidade do sono dos cuidadores de idosos dependentes de cadeira de rodas com diagnóstico de demência vascular e mista.

\section{Referências}

1. Winter DA. Human balance and posture control during standing and walking. Gait Posture. 1995;3:193214. doi:10.1016/0966-6362(95)99069-W.

2. Ruchinskas RA, Singer, HK, Repetz N. Cognitive status and ambulation in geriatric rehabilition: walking without thinking? Arch Phys Med Rehabil. 2000;81:1224-8. doi:10.1053/apmr.2000.6976.

3. Waite LM, Grayson DA, Piguet $\mathrm{O}$, Creasey H, Bennett HP, Broe GA. Gait slowing as a predictor of incident dementia: 6-year longitudinal data from the Sydney Older Persons Study. J Neurol Sci. 2005;15:229:89-93. doi:10.1016/j.jns.2004.11.009.

4. Almeida OP. Manejo dos distúrbios de comportamento em pacientes demenciado. In: Forlenza OV, Almeida OP. Depressão e demência no idoso: tratamento psicológico e farmacológico. São Paulo: Ed. Lemos; 1997. p. 152-166.

5. Peters KR, Rockwood K, Black SE, Bouchard R, Gauthier S, Hogan D, et al. Characterizing neuropsychiatric symptoms in subjects referred to dementia clinics. Neurology. 2006;66:523-8. doi:10.1212/01. wnl.0000198255.84842.06.

6. Tran P, Schimidt K, Gallo J, Tuppo E, Scheinthal S, Chopra A, et al. Neuropsychiatric symptoms and medical illness in patients with dementia: an exploratory study. J Am Osteopath Assoc. 2006;106:412-4. PMid:16912340.

7. André C. Demência vascular: dificuldades diagnósticas e tratamento. Arq Neuropsiquiatr. 1998;56:498-510. doi:10.1590/S0004-282X1998000300025.

8. American Psychiatric Association (APA). Manual diagnóstico e estatístico de transtornos mentais: DSM-IV-TR. 4. ed. Porto Alegre: Artmed; 2002. p. 168-77. 
9. Román GC, Tatemichi TK, Erkinjuntti T, Cummings JL, Masdeu JC. Vascular dementia: diagnostic criteria for research studies. Report of the NINCDS-AIREN internacional workshop. Neurology. 1993;43:250-60. doi:10.1212/WNL.43.2.250.

10. Longstreth WT Junior, Manolio TA, Arnold A. Clinical correlates of white matter findings on cranial magnetic resonance imaging of 3301 elderly people. The Cardiovascular Health Study. Stroke. 1996;27:127482. doi:10.1161/01.STR.27.8.1274.

11. Langa KM, Foster NL, Larson EB. Mixed dementia: emerging concepts and therapeutic implications. JAMA. 2004;15:2901-08. doi:10.1001/jama. 292.23.2901.

12. Zekry D, Duyckaerts C, Hauw JJ. Mixed dementia: a neuropathologic point of view. Psychol Neuropsychiatr Vieil. 2005;3:251-9. PMid:16316816.

13. Kalaria, RN, Ballard C. Overlap between pathology of the Alzheimer disease and vascular dementia. Alzheimer Dis Assoc Disord.1999;13:S115-23. doi: 10.1097/00002093-199912003-00017.

14. Bullock R. The needs of the caregiver in the long-term treatment of Alzheimer disease. Alzheimer Dis Assoc Disord. 2004;18:S17-S23. doi:10.1097/01. wad.0000127493.65032.9a.

15. Ory MG, Hoffman RR, Yee JL, Tennstedt S, Schulz R. Prevalence and impact of caregiving: a detailed comparison between dementia and nondementia caregivers. Gerontologist.1999;39:177-85. doi:10.1093/ geront/39.2.177.

16. Roth M, Tym E, Mountjoy CQ, Huppert FA, Hendrie H, Verma S, et al. CAMDEX a standardized instrument fot the diagnosis of mental disorders in the elderly with special reference to the early detection of dementia. Br J Psychiatry.1986;149:698-709. doi:10.1192/ bjp.149.6.698.

17. Cummings JL, Mega M, Gray K, Rosenberg-Thompson S, Carusi DA, Gorbein J. The Neuropsychiatric Inventory: comprehensive assessment of psychopathology in dementia. Neurology. 1994;44:2308-14. doi:10.1212/ WNL.44.12.2308.
18. Zomer J, Peied AH, Rubin E, Lavie P. Mini-sleep Questionnaire (MSQ) for screening large populations for EDS complaints. Sleep'84: Proceedings of the $7^{\text {th }}$ European Congress on Sleep Research. 1985. p. 467-470.

19. Engelborghs S, Maertens K, Mariën P, Vloeberghs E, Somers N, Nagels G, et al. Behavioural and neuropsychological correlates of frontal lobe features in dementia. Psychol Med. 2006;36(8):1173-82. doi: 10.1017/S003329170600777X.

20. Mungas D, Jagust WJ, Reed BR, Kramer JH, Weiner MW, Schuff N, et al. MRI predictors of cognition is subcortical ischemic vascular disease and Alzheimer's disease. Neurology. 2001;57(12):2229-35. doi:10.1212/ WNL.57.12.2229.

21. Grossman H, Bergamann C, Parker S. Dementia: a brief review. Mt Sinai J Med. 2006;73:985-92. PMid: 17195884.

22. Mori E. Functional brain imaging as a surrogate marker in vascular cognitive impairment. International Psychogeriatrics. 2003;15(Suppl 1):267-72. doi: $10.1017 /$ S1041610203009311.

23. Sbin ILS, Carter M, Masterman D, Fairbanks L, Cummings JL. Neuropsychiatric symptoms and quality of life in Alzheimer disease. Am J Geriatric Psychiatry. 2005;13(6):469-74. doi:10.1176/appi.ajgp.13.6.469.

24. McCurry SM, Vitiello MV, Gibbons LE, Logsdon RG, Teri L. Factors associated with caregiver reports of sleep disturbances in persons with dementia. A J Geriatric Psychiatry. 2006;14(2):112-20. doi:10.1097/01.JGP. 0000192499.25940.da.

25. Waite LM, Grayson DA, Piguet O, Creasey H, Bennett HP, Broe GA. Gait slowing as a predictor of incident dementia: 6-year longitudinal data from the Sydney Older Persons Study. J Neurol Sci. 2005;15:229:89-93. doi:10.1016/j.jns.2004.11.009.

Recebido: 22/01/2012 Received: 01/22/2012

Aprovado: $14 / 08 / 2012$ Approved: 08/14/2012 